\title{
Nuklearmedizin - Rising or falling star?
}

\author{
Hans-Jürgen Gallowitsch
}

(C) Springer-Verlag GmbH Austria, ein Teil von Springer Nature 2018

Die funktionelle und molekulare Bildgebung ist seit jeher eine Domäne der Nuklearmedizin und wurde durch technische Innovationen, vor allem im Rahmen der Hybriddiagnostik wie SPECT/CT, PET/CT und PET/MR, enorm aufgewertet, was durchaus auch Begehrlichkeiten von mancher Seite weckt. Wenngleich eine gewisse Schnittmenge zu anderen Fachrichtungen gegeben ist, wie innere Medizin/Endokrinologie und Radiologie, ist das herausragende Merkmal, welches das klinische Fach Nuklearmedizin von den anderen Fachrichtungen abgrenzt, die Möglichkeit, zugrundeliegende Stoffwechselprozesse abzubilden sowie die zunehmende Anzahl diverser Radiopharmazeutika, welche Stoffwechselvorgänge, Rezeptorbindungen, entzündliche oder immunologische Prozesse sichtbar machen und den Einsatz kombinierter diagnostischer/therapeutischer Systeme, den Theranostics, ermöglicht.

Das älteste Theranostikum stellt J-131 dar, welches im Rahmen der Radiojodganzkörperszintigraphie und der Radiojodtherapie auch heute noch routinemäßig zum Einsatz kommt. Auch Metajodbenzylguanidin (MIBG) wird seit Jahrzehnten sowohl diagnostisch (J-123 MIBG) als auch therapeutisch (J-131 MIBG z.B. Phäochromozytom, Neuroblastom) genutzt. Darüber hinaus werden zunehmend Theranostika für den Einsatz bei Neuroendokrinen Tumoren (Ga-68 DOTANOC/TOC - Lu-177 Dotatate/Y-90 DOTATOC), für das Prostatakarzinom (Ga-68 PSMA/Lu-177 PSMA) zur zielgerichteten Diagnostik und Therapie dieser Tumore eingesetzt. Andere Theranostika sind aktuell Gegenstand klinisch-wissenschaftlicher Studien

Univ.-Doz. Dr. H.-J. Gallowitsch, EOA $(\bowtie)$

Abteilung für Nuklearmedizin und Endokrinologie, PET-CT Center, Klinikum Klagenfurt am Wörthersee, Feschnigstraße 11, 9020 Klagenfurt, Österreich hans.gallowitsch@kabeg.at und werden in naher Zukunft für den Einsatz am Menschen verfügbar sein.

Das aktuelle Themenheft zeigt anhand des Prostatakarzinoms das Funktionieren dieses theranostischen Konzeptes auf. Grundlage ist ein gegen das Prostata-spezifische Membranantigen (PSMA) gerichteter Tracer, der mittels Markierung mit einem diagnostischen (Ga-68 oder Cu-64 - Positronenemitter) oder therapeutischen (Lu-177 - Betastrahler) Radionuklid spezifisch Tumorherde visualisieren und auch therapieren kann. Bei insgesamt geringer Toxizität (deutlich geringer als zum Einsatz kommende Chemotherapeutika) können somit simultan Knochenläsionen, Lymphknoten-, aber auch Organmetastasen behandelt werden.

In dem Übersichtsartikel von Christian Uprimny wird der aktuelle Stellenwert der Ga-68 PSMA PET/CT in der Primärdiagnose, Staging, Therapiekontrolle und Restaging beleuchtet. Trotz zahlreicher Studien, welche den Wert dieser Methode belegen, hat diese Untersuchung aufgrund unzureichender prospektiver Studien im Vergleich zum Goldstandard, der histopathologischen Untersuchung, noch nicht Eingang in die Leitlinien gefunden. Der Autor versucht, anhand der bisherigen Daten Empfehlungen für den möglichen Einsatz dieser Methode zu geben.

Siroos Mirzaei weist in einer kurzen Übersicht anschaulich anhand einiger Fallbeispiele auf den Wert auch alternativer Tracer wie F-18 Cholin und Cu-64 PSMA hin. Durch die kurze Halbwertszeit von Ga68 (60 min) ist eine Versorgung von Zentren über eine längere Distanz nicht möglich. Mit der Entwicklung F-18 markierter Tracer wird in Zukunft aufgrund der längeren Halbwertszeit von F-18 (2h) dieser Tracer auch für PET-CT Zentren verfügbar sein, welche keinen Zyklotron vor Ort oder in unmittelbarer Nähe aufweisen. Auch Tc-99 markierbare PSMA 
Tracer für Zentren ohne PET/CT sind bereits verfügbar.

Die diagnostische Bildgebung spielt auch eine wesentliche Rolle in der Primärdiagnostik, dem Staging und Restaging von Neuroblastomen, Phäochromozytomen und Paragangliomen. Während die konventionelle Schnittbilddiagnostik eine hohe Sensitivität aufweist, ist die Spezifität eher bescheiden. Infolge der Heterogenität der Tumoren bzgl. Metastasierungsrisiko, Biochemie und Genetik wird ein differenziertes Vorgehen je nach Tumorentität erforderlich. Mit den konventionellen nuklearmedizinischen Tracern J-123 MIBG und In-111 Octreotide sowie den PET-Tracern F-18 FDG, F-18 DOPA und Ga-68 markierten Somatostatin-Analoga steht dafür ein großzügiges Instru- mentarium zur Verfügung. Alexander Kroiss beleuchtet in seiner Übersicht den differenzierten Einsatz dieser Tracer bei diesen Tumorentitäten.

Die zunehmende Implementierung der PET/CT in die Leitlinien unterschiedlichster onkologischer Erkrankungen, die ständig ansteigende Zuweisungsfrequenz zur funktionellen Diagnostik sowie der Bedarf an nebenwirkungsarmer zielgerichteter, Tumorund Patienten-spezifischer Therapie lässt die Nuklearmedizin als Fixstern mit Zukunftspotential im medizinisch-diagnostisch/therapeutischen Kosmos aufleuchten.

Interessenkonflikt H.-J. Gallowitsch gibt an, dass kein Interessenkonflikt besteht. 